\title{
ACTIVIDAD ANTIOXIDANTE Y FOTOPROTECTORA in vitro DE UNA LOCIÓN Y GEL ELABORADOS CON EXTRACTO ESTABILIZADO DE CAMU CAMU (Myrciaria dubia Kunth.)
}

\author{
Miguel Ángel Inocente Camones ${ }^{1 *}$, Gloria Eva Tomas Chota ${ }^{3}$, Juana Huamán Malla ${ }^{3}$, \\ Ana María Muñoz Jáuregui ${ }^{4}$, Rosa Isabel García Morán ${ }^{2}$, Glorinda Quispe Fuentes ${ }^{2}$, \\ Christian Jesús Palomino Pacheco ${ }^{1}$, Evelyng Del Rosario Taype Espinoza ${ }^{2}$.
}

\begin{abstract}
RESUMEN
Esta investigación tuvo como finalidad evaluar la capacidad antioxidante y fotoprotectora de una loción y un gel elaborados con extracto estabilizado de los frutos de camu camu (Myrciaria dubia Kunth). Se realizó los controles de calidad fisicoquímica, microbiológica y estabilidad a condiciones normales y aceleradas, lo cual permitió elaborar parámetros iniciales para los protectores solares con extracto de camu camu. Se determinó la actividad antioxidante por el método de DPPH y ABTS, valores de 876,729 umol Trolox/g camu camu para el gel y 1389,650 umol Trolox/g camu camu para la loción (método DPPH) y valores de 15,330 mmol Trolox/g camu camu para el gel y $23.384 \mathrm{mmol}$ Trolox/g camu camu para la loción (método ABTS). El FPS de las formulaciones se determinó mediante un método in vitro desarrollado por Mansur. Se obtuvo valores de 10,897 $\pm 0,298$ para el gel y 13,401 $\pm 0,319$ para la loción.
\end{abstract}

Palabras clave: Myrciaria dubia Kunth., capacidad fotoprotectora, capacidad antioxidante, loción, gel, extracto estabilizado.

\section{ANTIOXIDANT ACTIVITY AND PHOTOPROTECTIVE in vitro OF LOTION AND GEL PROCESSED WITH EXTRACT STABILIZED OF CAMU CAMU (Myrciaria dubia Kunth.)}

\footnotetext{
ABSTRACT

In this research was to evaluated the antioxidant and photoprotective capacity of a lotion and a gel made with stabilized extract of the fruits of camu camu (Myrciaria dubia Kunth). Quality controls physicochemical, microbiological and stability to normal and accelerated conditions were performed, which allowed to develop initial parameters sunscreens extract of camu camu. Antioxidant activity by DPPH and ABTS method, values of 876.729 umol Trolox/g camu camu for gel and $1389.650 \mathrm{umol}$ Trolox/g camu camu for lotion was determined (DPPH method) and values of $15.330 \mathrm{mmol}$ Trolox/g camu camu for gel and $23.384 \mathrm{mmol}$ Trolox/g

Maestría de productos Naturales y Biocomercio. Facultad de Farmacia y Bioquímica. UNMSM.

AYRU COSMETIC SAC. Departamento de Investigación y Desarrollo. Av. Gerardo Unger 6322. Urb. Santa Luzmila. Comas.

3 Laboratorio de Productos Naturales. Facultad de Química e Ingeniería Química. UNMSM. Av. Germán Amézaga 375. Cercado de Lima. Lima. Perú.

4 Escuela Académico Profesional de Ciencias de los Alimentos. Facultad de Farmacia y Bioquímica. UNMSM.

minocente@farmaceuticos.com, investigacionayru@hotmail.com
} 
camu camu for lotion (ABTS method). The SPF of the formulations was determined by an in vitro method developed by Mansur. $10.897 \pm 0.298$ values were obtained for the gel and $13.401 \pm 0.319$ for lotion.

Keywords: Myrciaria dubia Kunth, photoprotective capacity, antioxidant capacity, lotion, gel, stabilized extract. 6322. Urb. Santa Luzmila. Comas.

\section{INTRODUCCIÓN}

Se ha desarrollado numerosos estudios sobre el efecto de los antioxidantes en la prevención del eritema cutáneo, aunque el espectro de acción de los antioxidantes se superpone con el espectro de acción de la radiación UVA $(320-400 \mathrm{~nm})$, por lo que la eficacia fotoprotectora de los mismos no sólo vendrá determinada por la protección frente al eritema fotoinducido, sino también por la protección sobre los daños moleculares y sobre el ADN derivados del estrés oxidativo generado por la radiación UV. La sensibilidad del mundo científico al daño generado por el estrés oxidativo en todos los ámbitos de la salud y el hecho de que la radiación ultravioleta sea el agente más eficaz en la producción de daño cutáneo por estrés oxidativo, hace que actualmente se esté en una búsqueda permanente de sustancias antioxidantes que actúen en este ámbito tanto por vía tópica como sistémica. ${ }^{1}$

Según la normativa de la Comunidad Andina. Decisión 516, artículo 21; se puede utilizar sustancias nuevas de origen subregional que no se encuentren consideradas en los listados internacionales reconocidos, siempre y cuando un país miembro apruebe su comercialización e informe de este hecho a los demás países miembros. De esta manera se impulsa un proceso de investigación y desarrollo basado en el uso sostenible de la inmensa diversidad biológica de los países andinos ${ }^{2}$.

Racan et al. ${ }^{3}$ desarrollaron un protector solar con líquenes ricos en ácido úsnico, con un FPS 3,4. Así, también analizaron la capacidad fotoprotectora del Peumus boldus y de los compuestos aromáticos de líquenes chilenos que viven en las áreas de alta incidencia de radiación ultravioleta y encontraron FPS máximo de 10 para las formulaciones producidas con los líquenes y FPS igual a 3.4 para la formulación con Peumus boldus. Tabriz et al ${ }^{4}$, analizaron el FPS de lociones fotoprotectoras con el extracto de flor de Rosa damascena que presenta flavonoides en su composición. Souza et $a l^{5}$, estudiaron los extractos de las flores y hojas de Achillea millefolium, planta que presenta flavonoides; sin embargo, no fueron efectivos en la preparación de un producto fotoprotector. Nascimento S. et al ${ }^{6}$, estudiaron el incremento del FPS en formulaciones fotoprotectoras utilizando extractos de própolis verde y rojo, demostrando que se produce un efecto sinérgico de estos extractos con el filtro sintético empleado en una formulación, lo que se traduce en una mayor protección solar. Velasco et al. ${ }^{7}$ evaluaron la eficacia fotoprotectora de rutina con filtros sintéticos como poctilmetoxicinamato y bezofenona-3, llegando a la conclusión que existe sinergismo en la elevación del FPS cuando este flavonoide es asociado con filtros sintéticos en concentraciones mínimas. Casagrande et al. ${ }^{8}$ estudiaron la quercetina, flavonoide que posee alta actividad antioxidante entre los flavonoides; demostraron que formulaciones tópicas conteniendo quercetina inhiben satisfactoriamente el daño cutáneo inducido por la radiación UVB en ratones.

Otros autores como Aquino et al. ${ }^{9}$ evaluaron la actividad antioxidante in vitro y la actividad fotoprotectora in vivo de los extractos etanólicos de Culcitium reflexun que demostraron marcada efectividad como antioxidantes y fotoprotectores. Sustancias como los flavonoides del tipo kaempferol y derivados de la quercetina e hidroxicinamatos como ácido cafeico, ácido ferúlico, ácido p-cumárico y ácido cinámico fueron relacionados en el estudio realizado por Bonina et $a l .^{10}$ al demostrar la actividad antioxidante y fotoprotectora de extractos 
metanólicos liofilizados de Capparis spinosa. Estudios en Brunilla vulgaris y su principal componente fenólico, el ácido rosmarínico, realizados por Psotova et al. ${ }^{11}$, demostraron el efecto fotoprotector del extracto dependiente de la concentración de ésta contra los cambios producidos en células queratinocíticas humanas inducidas por radiación UVA.

Los frutos de camu camu destacan por su contenido en compuestos fenólicos y vitamina $C .{ }^{12,13}$ En este contexto, los frutos de Myrciaria dubia Kunth. representan una fuente vegetal con activos potenciales para ser usados como coadyuvante de los fotoprotectores solares, debido a su contenido de antioxidantes.

En el Perú existen escasos estudios sobre productos naturales que puedan usarse como fotoprotectores en formulaciones cosméticas. Teniendo en cuenta que el Perú es un país de extraordinaria variedad de recursos naturales, se debe aprovechar la oportunidad de desarrollar productos con valor agregado, tales como fotoprotectores naturales, que puedan brindar efectos benéficos o preventivos frente a enfermedades que puedan estar relacionadas con el exceso de exposición a la radiación solar.

\section{Lugar de ejecución}

\section{PARTE EXPERIMENTAL}

El presente trabajo de investigación se realizó en el Área de Investigación y Desarrollo de AYRU COSMETIC SAC y el laboratorio de Productos Naturales de la Facultad de Química e Ingeniería Química de la Universidad Nacional Mayor de San Marcos.

\section{Muestra}

El fruto de camu camu (Myrciaria dubia Kunth) fue colectado en la ciudad de Pucallpa, provincia Coronel Portillo, departamento de Ucayali entre los 150 y $200 \mathrm{msnm}$ en el mes de enero del 2013. Se realizó la selección de frutos en buen estado, con un estado de madurez intermedio (pintonas).

\section{Reactivos}

Etanol absoluto QP, metanol QP, agua destilada, DPPH (2,2-difenil-1-picrilhidrazil), ABTS ácido 2,2'-azinobis-(3-etibenzotiazolin-6-sulfónico), persulfato de sodio, trolox (6-hidroxi-2, 5, 7,8 tetrametilcromo-2 ácido carboxílico 97\%) Sigma, agua desionizada.

\section{Equipos}

Espectrofotómetro UV-visible (Thermo Scientific), balanza analítica de capacidad de $1 \mathrm{mg}$ $200 \mathrm{~g}$ (Ohaus Pioneer), pHmetro digital HANNA HI 8424, balanza electrónica $1 \mathrm{mg}-200 \mathrm{~g}$ (OHAUS Pioneer), baño María.

\section{Métodos de análisis}

\section{Obtención y tratamiento del extracto de camu camu estabilizado}

Se elaboró un extracto de camu camu estabilizado. Los frutos de camu camu fueron seleccionados para descartar la materia prima dañada que presente signos de putrefacción, partes blandas y contaminación visible. Los frutos fueron lavados y desinfectados. Luego se licuó los frutos de camu camu con cubos de hielo y una solución de ácido cítrico (5:1). Se agregó una mezcla de agentes viscosantes (AYRUEXT $21{ }^{\circledR}$ ) con el extracto de camu camu (1:2) y se dejó reposar en frío $8^{\circ} \mathrm{C}$ por 1 hora. Luego se licuó y filtró sobre una tela de nylon.

\section{Desarrollo de la loción y el gel con extracto estabilizado de camu camu Pre-formulación}

Para la elección de la forma farmacéutica tópica y excipientes se tomó en cuenta algunos criterios, tales como factibilidad de fabricación, compatibilidad con el extracto, aspecto, 
costo, estabilidad y seguridad. Tomando en cuenta estos criterios y en base a las características de formas farmacéuticas tópicas se eligió las formas de loción y gel para el desarrollo de las formulaciones.

\section{Elaboración de formulaciones ${ }^{14,15}$}

Se realizó ensayos de formulaciones conteniendo el $15 \%$ de los extractos en el producto final. Así también, se ensayó métodos de incorporación de los extractos en frío. Se realizó formulaciones por cada tipo de forma farmacéutica: lociones y geles conteniendo los extractos y filtros sintéticos, para evaluar posible sinergia entre los mismos. Las tablas 1 y 2 muestran la composición final obtenida de la loción y gel, así como los placebos utilizados.

Tabla 1. Composición cuali-cuantitativa de las lociones.

\begin{tabular}{|c|c|c|}
\hline PRODUCTO & COMPOSICIÓN (MUESTRA) & COMPOSICIÓN (PLACEBO) \\
\hline \multirow{5}{*}{$\begin{array}{l}\text { Loción con } 15 \% \text { de extracto } \\
\text { de camu camu estabilizado y } \\
\text { filtro solar }\end{array}$} & Extracto de camu camu $15 \%$ & Filtro solar: Benzofenona 4 \\
\hline & Filtro solar: Benzofenona 4 & Preservantes: Ácido sórbico \\
\hline & Preservantes: Ácido sórbico & Excipientes: CMC, propilenglicol, \\
\hline & Excipientes: CMC, propilenglicol, etanol & etanol $20^{\circ}$, fragancia, agua \\
\hline & $20^{\circ}$, fragancia, agua & \\
\hline \multirow{4}{*}{$\begin{array}{l}\text { Loción con } 15 \% \text { de extracto } \\
\text { de camu camu estabilizado }\end{array}$} & Extracto de camu camu $15 \%$ & Preservantes: Ácido sórbico \\
\hline & Preservantes: Ácido sórbico & \multirow{3}{*}{$\begin{array}{l}\text { Excipientes: CMC, propilenglicol, } \\
\text { etanol } 20^{\circ} \text {, fragancia, agua }\end{array}$} \\
\hline & Excipientes: CMC, propilenglicol, etanol & \\
\hline & $20^{\circ}$, fragancia, agua & \\
\hline
\end{tabular}

Tabla 2. Composición cuali-cuantitativa de geles.

\begin{tabular}{lll}
\hline PRODUCTO & COMPOSICIÓN (MUESTRA) & COMPOSICIÓN (PLACEBO) \\
\hline $\begin{array}{l}\text { Gel con 15\% de extracto de } \\
\text { filtro solar }\end{array}$ & Extracto de camu camu 15\% & Filtro solar: Benzofenona 4 \\
& Filtro solar: Benzofenona 4 & Preservantes: Ácido sórbico \\
& Preservantes: Ácido sórbico & Excipientes: CMC, propilenglicol, \\
& Excipientes: CMC, propilenglicol, etanol & etanol 20 , fragancia, agua \\
\hline Gel con 15\% de extracto de & Extracto de camu camu 15\% & \\
camu camu estabilizado & Preservantes: Ácido sórbico & Preservantes: Ácido sórbico \\
& Excipientes: CMC, propilenglicol, etanol & Excipientes: CMC, propilenglicol, \\
& $20^{\circ}$, fragancia, agua & \\
\hline
\end{tabular}




\section{Evaluación de la calidad de la loción y gel con filtro y extracto de camu camu Análisis fisicoquímico y sensorial de la loción y el gel}

Se realizó el análisis sensorial y fisicoquímico de la loción y gel tomando en cuenta el aspecto, color y olor. El $\mathrm{pH}$ y viscosidad fueron determinados por MICROBIOL SA.

\section{Análisis microbiológico de la loción y el gel}

El análisis microbiológico fue determinado por MICROBIOL SA. Las especificaciones como límite máximo se han considerado en referencia a la Secretaría General de la Comunidad Andina, según Resolución 1482. Se ha determinado cantidad de microorganismos aerobios mesófilos, mohos y levaduras como mínimo $\leq 50 \times 10^{2} \mathrm{ufc} / \mathrm{g}$ y ausencia de $S$. aureus, $P$. aeruginosa y E. coli.

\section{Evaluación de la estabilidad acelerada de la loción y el gel.}

La evaluación de la estabilidad acelerada de la loción y el gel ha sido determinada por MICROBIOL SA. La evaluación se ha determinado en condiciones normales a $30^{\circ} \mathrm{C} \pm 2^{\circ} \mathrm{C} \mathrm{y}$ $65 \% \pm 5 \%$ HR y condiciones aceleradas $40^{\circ} \mathrm{C} \pm 2^{\circ} \mathrm{C}$ y $75 \% \pm 5 \% \mathrm{HR}$, Se evalúo el análisis organoléptico (aspecto, color, olor y consistencia), análisis fisicoquímico ( $\mathrm{pH}$ y viscosidad a $25^{\circ} \mathrm{C}$ ) y análisis microbiológico (microrganismos aerobios mesófilos, hongos filamentosos y levaduras ufc/g).

\section{Determinación de la actividad antioxidante}

La actividad antioxidante de la loción y gel se determinó por el método 2,2-difenil-1picrilhidracilo (DPPH) y el método ácido 2,2'-azinobis-(3-etibenzotiazolín-6-sulfónico) (ABTS) ${ }^{16,17}$.

\section{Evaluación de la actividad antioxidante mediante el método DPPH}

En una batería de 3 tubos se colocaron $0,1 \mathrm{~mL}$ de la loción, gel y Trolox, se le adicionó 3,9 mL de una solución de DPPH 0,1 mM, se homogenizó y dejó en reposo durante 30 minutos a temperatura ambiente y bajo oscuridad. Se midió la absorbancia a $517 \mathrm{~nm}$ en un espectrofotómetro UV-Vis. Todos los análisis fueron realizados por triplicado $(n=3)$.

El porcentaje de inhibición o porcentaje de decoloración fue calculado como:

$$
\text { \% inhibición DPPH }=100(\mathrm{~A}-\mathrm{B}) / \mathrm{A}
$$

Donde: A: Lectura de absorbancia del DPPH

B: Lectura de absorbancia de la muestra, después de $30 \mathrm{~min}$.

La concentración requerida para el 50\% de inhibición del radical libre DPPH (IC) fue calculada mediante la ecuación de la gráfica de concentración del producto y trolox vs \% inhibición. El blanco para calibrar el equipo fue metanol: agua (2:1). El blanco es DPPH en metanol 0,1 mM. Como control positivo se preparó una curva patrón de trolox a las mismas condiciones de análisis de la loción y gel, para comparar las IC. Los resultados se expresan en umol de Trolox/g de camu camu. Los resultados fueron procesados utilizando programas SPSS 21 y Microsoft Excel 2013. Todos los experimentos se realizaron por triplicado y expresados como valores promedio +/-DS.

\section{Evaluación de la actividad antioxidante mediante el método ABTS}

Para la segunda técnica el radical ABTS $+{ }^{18}$ sólo es estable máximo 2 días; por tal razón se ha preparado 2 litros solución stock del ácido 2,2'-Azinobis-(3-etibenzotiazolín-6-sulfónico) $(3,8 \mathrm{mg} / \mathrm{mL})$ con persulfato de sodio $(37,5 \mathrm{mg} / \mathrm{mL})$ a temperatura ambiente $\left(25^{\circ} \mathrm{C}\right)$. La mezcla se colocó en recipiente color ámbar y en oscuridad durante 16 horas a temperatura ambiente 
hasta lograr la estabilidad del reactivo. Posteriormente se tomó 0,5 mL de la solución madre de ABTS y se enrasó a $50 \mathrm{~mL}$ con etanol absoluto, y se lee a $754 \mathrm{~nm}$, se ajustó hasta obtener una

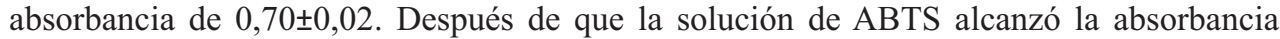
requerida, se tomó $3,9 \mathrm{~mL}$ de la solución y se mezcló con $0,1 \mathrm{~mL}$ de la loción y gel. Se homogenizó por 1 minuto y se midió la absorbancia a 7 minutos de reacción, a $754 \mathrm{~nm}$.

Se empleó las siguientes ecuaciones para obtener el \% de inhibición.

\% inhibición de ABTS $=\left[1-\left(\mathrm{A}_{2}-\mathrm{A}_{3}\right) / \mathrm{A}_{1}\right] 100$

Donde: $\quad \mathbf{A}_{1}=$ Absorbancia del ABTS en etanol

$\mathbf{A}_{2}=$ Absorbancia de la muestra a los 7 minutos

$\mathbf{A}_{3}=$ Absorbancia del blanco de muestra

La concentración requerida para el 50\% de inhibición del radical libre ABTS (IC) fue calculada mediante la ecuación de la gráfica de concentración del extracto y trolox vs $\%$ inhibición. El blanco para calibrar el equipo fue metanol: agua (2:1). El blanco es ABTS en metanol 0,1 mM. Como control positivo se preparó una curva patrón de trolox a las mismas condiciones de análisis de la loción y gel, para comparar las IC. Los resultados se expresan en mmol de Trolox/g de producto. Los resultados fueron procesados utilizando programas SPSS 21 y Microsoft Excel 2013. Todos los experimentos se realizaron por triplicado y los resultados expresados como valores promedio +/- DS.

\section{Determinación in vitro del Factor de Protección Solar (FPS) de la loción y gel.}

El Factor de Protección Solar (FPS) de la loción y gel elaboradas se determinó siguiendo la metodología in vitro descrita por Mansur et al. ${ }^{19}$ Éste análisis consiste en un método espectrofotométrico en el cual la formulación se diluye en etanol absoluto hasta una concentración de $0,2 \mathrm{mg} / \mathrm{mL}$, condición establecida por el autor para crear una correlación con el método in vivo. A través de la fórmula matemática desarrollada según el método, se relacionan los valores de absorbancia obtenidos de las muestras con el FPS de la formulación. El presente estudio evalúa el FPS en el rango de 290 a 320 nm (rango UVB), mas no en el rango UVAni UVC.

Para obtener las muestras diluidas de las formulaciones $(0,2 \mathrm{mg} / \mathrm{mL})$, se pesó $1,0 \mathrm{~g}$ de las formulaciones y se transfirieron a un matraz aforado de $100 \mathrm{~mL}$, se agrega $50 \mathrm{~mL}$ de etanol, se agita por 5 minutos, y luego se diluye a volumen con etanol; se homogeniza y luego se filtra descartando los primeros 10 mililitros. Una alícuota de 5,0 $\mathrm{mL}$ del filtrado se transfiere a un matraz aforado de $50 \mathrm{~mL}$ y se diluye a volumen con etanol. Luego una alícuota de 5,0 $\mathrm{mL}$ de la última dilución se transfiere a un matraz aforado de $25 \mathrm{~mL}$ y se lleva a volumen con etanol. Las absorbancias de las soluciones fueron determinadas en el rango de 290 a $320 \mathrm{~nm}$, con intervalos de $5 \mathrm{~nm}$ utilizando una cubeta de cuarzo de $1 \mathrm{~cm}$. Los análisis fueron realizados por triplicado y el FPS fue calculado de acuerdo con la ecuación desarrollada por Mansur et al. ${ }^{19}$ :

$$
\operatorname{FPS}=\mathrm{FC} \times \sum_{290}^{320} \times \operatorname{EE}(\lambda) \times \mathrm{I}(\lambda) \times \operatorname{Abs}(\lambda)
$$

Donde: FPS $=\quad$ Factor de Protección Solar

$\mathrm{FC}=10 \quad$ (factor de corrección)

$\mathrm{EE}(\lambda)=\quad$ Efecto eritemogénico de la radiación de longitud de onda $\lambda$

$\mathrm{I}(\lambda)=\quad$ Intensidad del sol en la longitud de onda $\lambda$

$\operatorname{Abs}(\lambda)=$ Absorbancia de la solución en la longitud de onda $\lambda$ 
La relación entre el efecto eritemogénico y la intensidad de la radiación de cada longitud de onda $(\operatorname{EE}(\boldsymbol{\lambda}) \times \mathrm{I}(\boldsymbol{\lambda}))$ es una constante determinada por Sayre et al. ${ }^{20}$ (tabla 3 )

Tabla 3. Constante determinada por Sayre et al $(\operatorname{EE}(\lambda) \times \mathrm{I}(\lambda)$.

\begin{tabular}{ccccccccr}
\hline Longitud de onda (nm) & $\mathbf{2 9 0}$ & $\mathbf{2 9 5}$ & $\mathbf{3 0 0}$ & $\mathbf{3 0 5}$ & $\mathbf{3 1 0}$ & $\mathbf{3 1 5}$ & $\mathbf{3 2 0}$ & TOTAL \\
\hline EE $(\boldsymbol{\lambda}) \mathbf{x}$ I ( $\boldsymbol{\lambda})$ & 0,0150 & 0,0817 & 0,2874 & 0,3278 & 0,1864 & 0,0839 & 0,0180 & 1,000 \\
\hline
\end{tabular}

Fuente: Sayre R, Desrochers D, Marlow E.

\section{RESULTADOS Y DISCUSIÓN}

De la evaluación de la calidad de la loción y gel con filtro y extracto de camu camu Del análisis fisicoquímico y sensorial de la loción y gel

Los resultados del pH de la loción y gel (tabla 4), se encuentran en un rango de 3,0 a 5,0, lo cual indica que se encuentran en condiciones favorables para la piel humana.

Los resultados de la viscosidad demuestran la condición física de los productos, y de acuerdo a ello se enmarca a estabilidad relacionado con la consistencia.

Tabla 4. Resultados del análisis fisicoquímico de los protectores solares

\begin{tabular}{lcc}
\hline \multicolumn{1}{c}{ Análisis fisicoquímico } & Gel & Loción \\
$\mathrm{pH}\left(25^{\circ} \mathrm{C}\right)$ & 3,38 & 5,04 \\
Viscosidad cps $\left(25^{\circ} \mathrm{C}\right)$ & 457,50 & 5,00 \\
\hline
\end{tabular}

\section{Del análisis microbiológico de la loción y gel}

Los productos han sido fabricado en condiciones sanitarias óptimas, demostrado con la ausencia de $S$. aureus, $P$. aeruginosa y $E$. coli. El límite máximo permitido para los aerobios mesófilos, mohos y levaduras es $\leq 50 \times 10^{2} \mathrm{ufc} / \mathrm{g}$. (tabla 5 )

Tabla $\mathbf{N}^{0}$ 5. Resultados del análisis microbiológico de los protectores solares

\begin{tabular}{lcc}
\hline \multicolumn{1}{c}{$\quad$ Análisis microbiológico } & Gel & Loción \\
Numeración de aerobios mesófilos ufc/g & $<10$ & $37 \times 10$ \\
Numeración de mohos ufc/g & $<10$ & $<10$ \\
Numeración de levaduras, ufc/g & $<10$ & $48 \times 10$ \\
Presencia de Staphylococcus aureus/g & Ausente & Ausente \\
Presencia de Pseudomonas aeruginosa/g & Ausente & Ausente \\
Presencia de Escherichia coli/g & Ausente & Ausente \\
\hline
\end{tabular}

De la evaluación de la estabilidad acelerada de la loción con filtro y extracto

En las tablas 6 y 7, se observa que la loción ha mantenido el perfil microbiológico debajo del límite permitido, a condiciones normales y condiciones aceleradas. 
En el caso de la viscosidad de la crema, se observa resultados parejos y equivalentes hasta el sexto mes de análisis. Para el caso del $\mathrm{pH}$, los valores disminuyen con el tiempo, debido a la reducida cantidad de excipientes de la fórmula.

Tabla 6. Análisis de estabilidad de la loción, en condiciones normales

\begin{tabular}{|c|c|c|c|}
\hline \multirow{2}{*}{ Parámetro } & \multicolumn{3}{|c|}{ Tiempo de evaluación } \\
\hline & Tiempo cero & $3^{\circ} \mathrm{mes}$ & $6^{0}$ mes \\
\hline \multicolumn{4}{|l|}{ Organolépticos } \\
\hline Color & $\begin{array}{l}\text { Anaranjado ligeramente } \\
\text { turbio }\end{array}$ & $\begin{array}{l}\text { Anaranjado ligeramente } \\
\text { turbio }\end{array}$ & $\begin{array}{c}\text { Anaranjado } \\
\text { ligeramente turbio }\end{array}$ \\
\hline Olor & Sui generis & Sui generis & Sui generis \\
\hline Aspecto & Homogéneo & Heterogéneo & Heterogéneo \\
\hline Consistencia & Líquida & Líquida & Líquida \\
\hline \multicolumn{4}{|l|}{ Fisicoquímicos } \\
\hline $\mathrm{pH}\left(25^{\circ} \mathrm{C}\right)$ & 5,04 & 4,24 & 4,78 \\
\hline Viscosidad $25^{\circ} \mathrm{C}$, cps & 5,00 & 5,00 & 5,00 \\
\hline \multicolumn{4}{|l|}{ Microbiológicos } \\
\hline Aerobios mesófilos, ufc/g & $37 \times 10$ & $<10$ & $<10$ \\
\hline Hongos filamentosos, ufc/g & $<10$ & $<10$ & $<10$ \\
\hline Levaduras, ufc/g & $48 \times 10$ & $<10$ & $<10$ \\
\hline
\end{tabular}

Tabla 7. Análisis de estabilidad de la loción, en condiciones aceleradas

\begin{tabular}{|c|c|c|c|}
\hline \multirow{2}{*}{ Parámetro } & \multicolumn{3}{|c|}{ Tiempo de evaluación } \\
\hline & Tiempo cero & $3^{\circ}$ mes & $6^{0}$ mes \\
\hline \multicolumn{4}{|l|}{ Organolépticos } \\
\hline Color & $\begin{array}{l}\text { Anaranjado ligeramente } \\
\text { turbio }\end{array}$ & $\begin{array}{l}\text { Anaranjado oscuro } \\
\text { ligeramente turbio }\end{array}$ & $\begin{array}{l}\text { Anaranjado oscuro } \\
\text { ligeramente turbio }\end{array}$ \\
\hline Olor & Sui generis & Sui generis & Sui generis \\
\hline Aspecto & Heterogéneo & Heterogéneo & Heterogéneo \\
\hline Consistencia & Líquida & Líquida & Líquida \\
\hline \multicolumn{4}{|l|}{ Fisicoquímicos } \\
\hline $\mathrm{pH}\left(25^{\circ} \mathrm{C}\right)$ & 5,68 & 5,20 & 4,83 \\
\hline Viscosidad $25^{\circ} \mathrm{C}$, cps & 5,00 & 5,00 & 5,00 \\
\hline \multicolumn{4}{|l|}{ Microbiológicos } \\
\hline Aerobios mesófilos, ufc/g & $<10$ & $<10$ & $<10$ \\
\hline Hongos filamentosos, ufc/g & $<10$ & $<10$ & $<10$ \\
\hline Levaduras, ufc/g & $<10$ & $<10$ & $<10$ \\
\hline
\end{tabular}

\section{De la evaluación de la estabilidad acelerada del gel con filtro y extracto}

En la tabla 8 y 9, se observa que el gel ha mantenido el perfil microbiológico debajo del límite permitido durante los 6 meses de análisis.

En el caso de la viscosidad del gel, se observa una disminución en el tercer mes de evaluación a condiciones normales y aceleradas; esto se debe a que la temperatura de $30^{\circ}$ a $40^{\circ} \mathrm{C}$ desfavorece la consistencia del producto, por lo cual la óptima temperatura sería de $25^{\circ} \mathrm{C}$. Para el caso del $\mathrm{pH}$, los valores disminuyen con el tiempo, debido a la reducida cantidad de excipientes de la fórmula. 
Tabla 8. Análisis de estabilidad del gel, en condiciones normales

\begin{tabular}{|c|c|c|c|}
\hline \multirow{2}{*}{ Parámetro } & \multicolumn{3}{|c|}{ Tiempo de evaluación } \\
\hline & Tiempo cero & $3^{\circ}$ mes & $6^{0}$ mes \\
\hline \multicolumn{4}{|l|}{ Organolépticos } \\
\hline Color & Amarillo turbio & Amarillo turbio & Amarillo turbio \\
\hline Olor & Sui generis & Sui generis & Sui generis \\
\hline Aspecto & Homogéneo & Heterogéneo & Heterogéneo \\
\hline Consistencia & Espesa & Ligeramente espesa & Ligeramente espesa \\
\hline \multicolumn{4}{|l|}{ Fisicoquímicos } \\
\hline $\mathrm{pH}\left(25^{\circ} \mathrm{C}\right)$ & 3,38 & 3,22 & 2,89 \\
\hline Viscosidad $25^{\circ} \mathrm{C}$, cps & 457,5 & 137,5 & 77,5 \\
\hline \multicolumn{4}{|l|}{ Microbiológicos } \\
\hline Aerobios mesófilos, ufc/g & $<10$ & $<10$ & $<10$ \\
\hline Hongos filamentosos, ufc/g & $<10$ & $<10$ & $<10$ \\
\hline Levaduras, ufc/g & $<10$ & $<10$ & $<10$ \\
\hline
\end{tabular}

Tabla 9. Análisis de estabilidad del gel, en condiciones aceleradas

\begin{tabular}{lccc}
\hline \multicolumn{1}{c}{ Parámetro } & Tiempo cero & $\begin{array}{c}\text { Tiempo de evaluación } \\
\mathbf{3}^{\mathbf{0}} \text { mes }\end{array}$ & $\mathbf{6}^{\mathbf{0}}$ mes \\
$\begin{array}{l}\text { Organolépticos } \\
\text { Color }\end{array}$ & Amarillo turbio & Amarillo oscuro turbio & $\begin{array}{c}\text { Amarillo oscuro } \\
\text { turbio } \\
\text { Sui generis }\end{array}$ \\
Olor & Sui generis & Sui generis & Heterogéneo \\
Aspecto & Heterogéne & Heterogéneo & Semilíquido \\
Consistencia & Espesa & Ligeramente espesa & \\
Fisicoquímicos & & & 2,87 \\
pH $\left(25^{\circ} \mathrm{C}\right)$ & 3,38 & 3,18 & 20,0 \\
Viscosidad $25^{\circ} \mathrm{C}, \mathrm{cps}$ & 140 & 57,5 & $<10$ \\
Microbiológicos & & $<10$ & $<10$ \\
Aerobios mesófilos, ufc/g & $<10$ & $<10$ & $<10$ \\
$\begin{array}{l}\text { Hongos filamentosos, ufc/g } \\
\text { Levaduras, ufc/g }\end{array}$ & $<10$ & $<10$ & \\
\hline
\end{tabular}

\section{De la actividad antioxidante}

De acuerdo al método DPPH, se obtuvieron valores expresados en umol Trolox/g camu camu de 876,729 para el gel con filtro y extracto y 1328,937 para el gel con extracto. En el caso de la loción se obtuvo 1389,650 para la loción con filtro y extracto, y 1657,625 para la loción con extracto. (figura 1). 


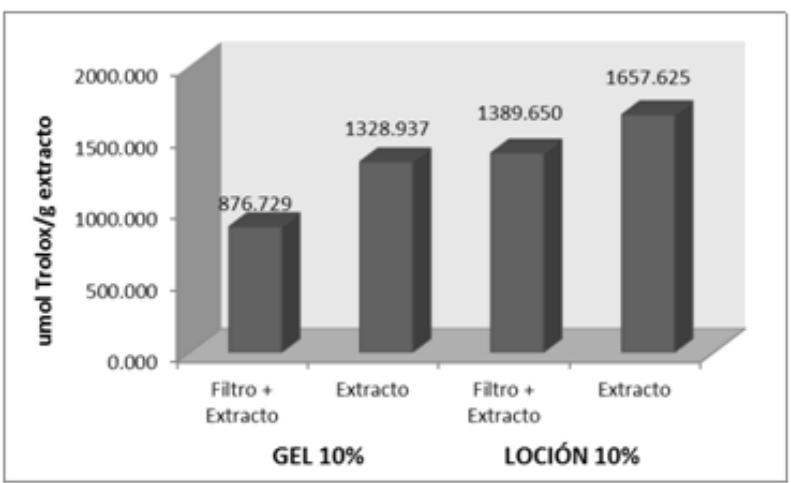

Figura 1. Comparación de la capacidad antioxidante, según método DPPH.

De acuerdo al método ABTS, se obtuvo valores expresados en mmol Trolox/g camu camu de 15,330 para el gel con filtro y extracto, y 16,667 para el gel con extracto. En el caso de la loción se obtuvo 23,384 para la loción con filtro y extracto y 30,516 para la loción con extracto. (figura 2).

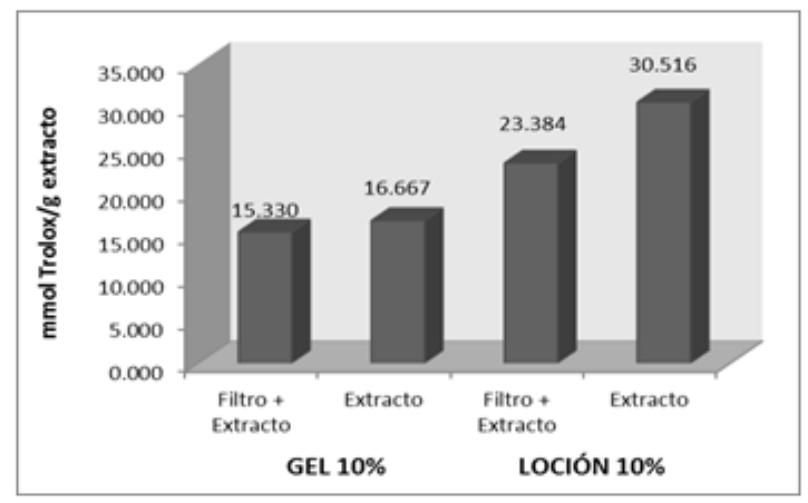

Figura 2. Comparación de la capacidad antioxidante, según método ABTS.

\section{De la determinación in vitro del Factor de Protección Solar (FPS).}

\section{Determinación del FPS de los geles}

Se obtuvieron valores de FPS de 0,834 $\pm 0,005$ para el gel placebo, 10,897 $\pm 0,298$ para el gel con filtro y extracto, $10,686 \pm 0,05$ para el gel con filtro solar y 1,510 $\pm 0,036$ para el gel con extracto (figura 3) 


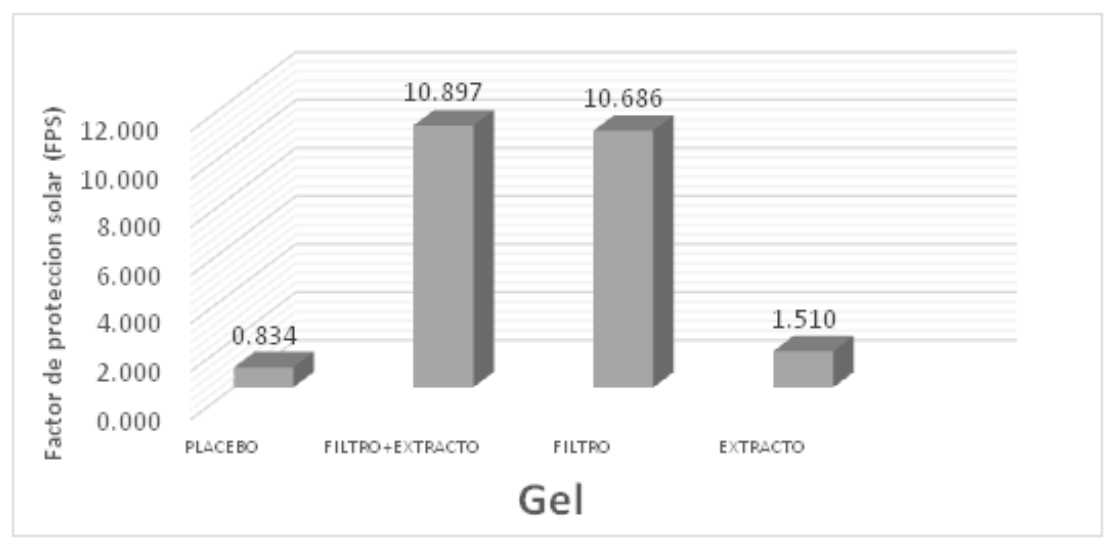

Figura 3. Valores de FPS en los geles.

\section{Determinación del FPS de las lociones}

Se obtuvieron valores de FPS de 0,589 $\pm 0,057$ para la loción placebo, 13,401 $\pm 0,319$ para la loción con filtro y extracto, $12,066 \pm 0,053$ para la loción con filtro solar y 2,667 $\pm 0,044$ para la loción con extracto (figura 4)

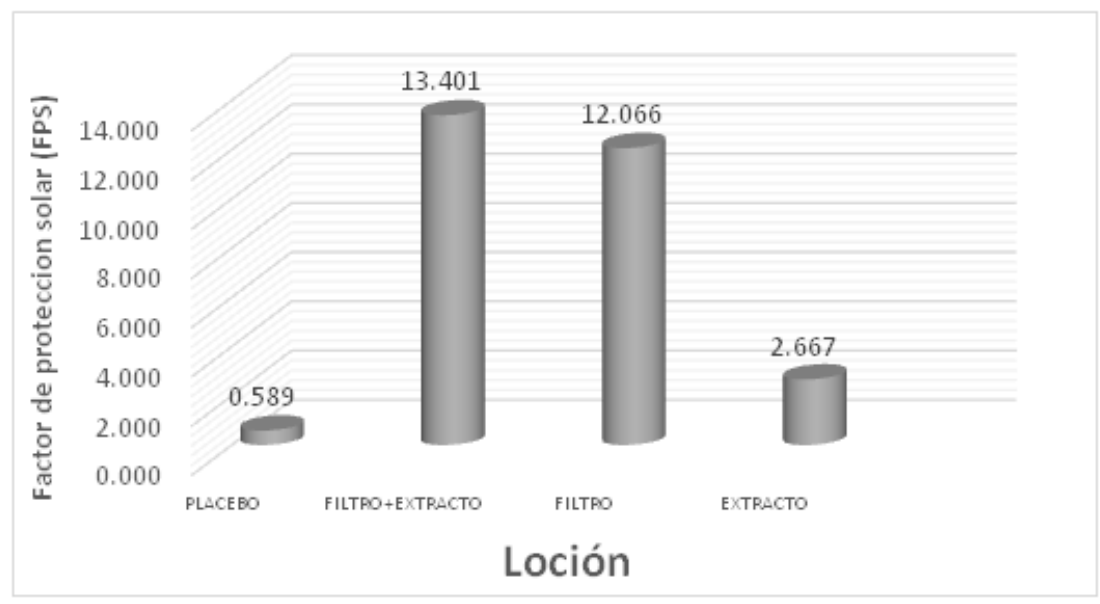

Figura 4. Valores de FPS en los lociones.

El fruto de camu camu (Myrciaria dubia HBK) brinda una excelente alternativa para ser utilizado como sinergista de los fotoprotectores en formulaciones cosméticas por poseer en su composición compuestos con actividad biológica, los que resaltan sustancias antioxidantes, como compuestos fenólicos y vitamina $\mathrm{C}^{12,13}$. 
Se eligió filtros solares comerciales más conocidos y disponibles en el mercado, además de ser ampliamente utilizados en fotoprotectores diversos y que tuvieran estudios científicos de eficacia frente a la protección contra las radiaciones UVA y UVB. Se utilizó el filtro de benzofenona 4 en los geles y lociones, ya que al ser soluble en agua permite su incorporación en productos hidrofílicos. La concentración de uso de estos filtros sintéticos se determinó tomando en cuenta los valores máximos permitidos de acuerdo a la FDA.

La asociación de los extractos y filtros solares sintéticos en las formulaciones, no presentaron elevación significativa del valor de SPF, que fue de 10,686 $\pm 0,05$ a 10,897 $\pm 0,298$ y de 12,066 $\pm 0,053$ a 13,401 $\pm 0,319$ para el gel. El último resultado demuestra que existe una posible sinergia significativa entre el extracto de camu camu con el filtro solar específicamente en la formulación de la loción. Sin embargo, las formulaciones con filtro solar denotan un valor de SPF alto cercano a SPF 15, especialmente para la loción. Estos resultados pueden explicarse tomando en cuenta que la eficacia de un filtro solar, es decir, la actividad fotoprotectora, depende de la capacidad de absorción de energía radiante atribuida a los grupos cromóforos, proporcional a su concentración, intervalo de absorción y longitud de onda donde ocurre la absorción máxima ${ }^{21}$. Al relacionar esta premisa con los resultados del presente estudio, se puede sugerir que los valores bajos de FPS es consecuencia a la baja concentración de las moléculas con capacidad para absorber la radiación UV (cromóforos). Adicionalmente, los valores bajos de FPS en las formulaciones con extractos, podrían relacionarse con el método utilizado para determinar la actividad fotoprotectora, ya que este método restringe la eficacia de las formulaciones elaboradas a los compuestos que absorben radiación sólo al rango UVB, 290 a 320nm, no incluyendo así a todo el rango UV; es decir desde los 200 hasta los $400 \mathrm{~nm}$.

\section{CONCLUSIONES}

Se estableció parámetros de la calidad fisicoquímica, microbiológica y estabilidad a condiciones normales y aceleradas de la loción y gel con extracto y filtro solar. Ambas formulaciones muestran actividad antioxidante con valores de 876,729 umol Trolox/g camu camu para el gel, y 1389,650 umol Trolox/g camu camu para la loción (método DPPH) y valores de 15,330 mmol Trolox/g camu camu para el gel, y 23,384 mmol Trolox/g camu camu para la loción (método ABTS). Las formas farmacéuticas de loción y gel conteniendo extracto estabilizado de camu camu y filtro solar presentan valores de Factores de Protección Solar (FPS) de 10,897 $\pm 0,298$ para el gel, y 13,401 $\pm 0,319$ para la loción. Se concluye que la loción y gel poseen propiedades antioxidantes y factor de protección solar acorde a las exigencias normativas.

\section{AGRADECIMIENTO}

Esta investigación se ha realizado gracias a la colaboración y asesoría del Laboratorio de Productos Naturales de la Facultad de Química e Ingeniería Química de la UNMSM y al financiamiento del FINCYT-FIDECOM.

\section{BIBLIOGRAFÍA}

1. De Gálvez MV. Antioxidantes en fotoprotección, ¿realmente funcionan? Actas Dermosifiliográficas. 2010; 101(3): 197-200.

2. Comisión de la Comunidad Andina. DECISION 516. Armonización de Legislaciones en materia de Productos Cosméticos. Gaceta Oficial del Acuerdo de Cartagena No. 771 del 14 de marzo de 2002.

3. Racan F, Rosan S, Boehm K, Fernandez E, Hidalgo M, Quihot W. et. al. Protection against UVB irradiation by natural filters extracted from lichens. J. Photochem. Photobiol. B. 2002; 68 (2-3): 133-9. 
4. Tabriz H, Mortazavi S, Kamalinejad M. An in vitro evaluation of various Rosa Damascena extracts as a natural antisolar agent. Int. J. Cosm. Sci. 2003; 25 (1): 259-265.

5. Souza T, Moreira R, Rangel V, Pietro R. Avaliação da atividade fotoprotetora de Achillea millefolium L. Rev. Bras. Farmacogn. 2005; 15 (1): 36-38.

6. Nascimento S, Nunes L, Lima A, Grangeiro Júnior S, Rolim. Incremento do FPS em formulação de protetor solar utilizando extratos de própolis verde e vermelha. Rev. Bras. Farm. 2009; 90 (4):334-339.

7. Velasco M, Balogh T, Pedriali C. Associação da Rutina com p-Metoxicinamato de Octila e Benzofenona-3: Avaliação in vitro da Eficácia Fotoprotetora por Espectrofotometria de Refletância. Lat. Am. J. Pharm. 2008; 27 (1):23-7.

8. Casagrande R, Georgetti S, Verri W. Protective effect of topical formulations containing quercetin against UVB-induced oxidative stress in hairless mice. J. Photochem. Photobiol. B. 2006; 84(1): 21-7.

9. Aquino R, Morelli S, Tomaino A, Pellegrino M, Saija A. Antioxidant and photoprotective activity of a crude extract of Culcitium reflexum H.B.K. leaves and their major flavonoids. Journal of Ethnopharmacology. 2001; 79(1): 183-191.

10. Bonina C, Puglia D, Ventura R, Aquino S, Tortora A, Sacchi A. et al. In vitro antioxidant and in vivo photoprotective effects of a lyophilizede extract of Cappariss pinosa L. buds. J. Cosmet. Sci. 2002; 53(6): 321-35.

11. Psotova J, Svobodova A, Kolarova H, Walterova D. Photoprotective properties of Prunella vulgaris and rosmarinic acid on human keratinocytes. J. Photochem. Photobiol. B. 2005; 84 (1): 167-174.

12. Villanueva, T.; Condezo-Hoyos, L.; Ramírez, E. Antocianinas, ácido ascórbico, polifenoles totales y actividad antioxidante en la cáscara de camu camu Myrciaria dubia (H.B.K.) Mc Vaugh. Revista Ciencia y Tecnología de Alimentos, Campinas. 2010. 30 (Supl. 1): 151-160 p.

13. Zamudio, L.B. 2007. Caracterização de Vitamina C em frutos de Camu-camu Myrciaria dubia (H.B.K.) em diferentes estágios de maturação do Banco Ativo de Germoplasma de Embrapa. Dissertação para Especialização em Nutrição Humana. Universidade de Brasília UnB Brasília DF - Brasil. 104 p.

14. Alcalde T. Alimentos Usados en Formulaciones Cosméticas. Propiedades y Aplicaciones. Offarm. 2007; 26 (1): 100-108.

15. Villarreal A. Formulación de una nanoemulsión dermocosmética, nutritiva y regeneradora de la piel. Tesis Maestría. Universidad De Los Andes, Facultad De Ciencias. Venezuela. 2004.

16. Antolovich M, Prenzler P, Patsalides E, MCDonald S, Robards K. Methods for testing antioxidant activity. Analystic. 2002; 127 (1): 183-198.

17. Friaa D. Kinetics of the reaction between the antioxidant Trolox and the free radical $\mathrm{DPPH}^{\circ}$ in semi-aqueous solution. Org. Biomol. Chem. 2006.

18. Re R., Pellegrini Ni., Proteggente A., Yang M., Rice Evans C. Antioxidant activity applying an improved ABTS radical cation decolorization assay. Free Radical Biology and Medicine; 1999; 26: 1231-1237.

19. Mansur J, Breder M, Mansur M, Azulay R. Determinação do fator de proteção solar por espectrofotometria. An. Bras. Dermatol. 1986; 61 (1): 121-124.

20. Sayre R, Desrochers D, Marlow E. Sunscreen testing methods: in vitro predictions of effectiveness. J. Soc. Cosmet. Chem. 1980; 31 (1): 133-43.

21. Silva Filho E, Sena G, Pires J. Moléculas inhibidoras de radiações UV. Cosmetic and Toiletries. 2003; 15 (1): 82-84. 